\title{
Pressure injury associated with the use of TALA. Patient case study taken in an advance unit: evaluation, diagnosis and nursing interventions
}

\begin{abstract}
Introduction: In Brazil, for example, pressure injuries characterize a serious public health problem.

Objective: To identify and characterize the factors that influenced the appearance of pressure injury in a trauma victim patient due to a motorcycle crash hospitalized at the Vilhena Regional Hospital using immobilization by splint for immobilization.
\end{abstract}

Discussion: The study evidenced that the number of LPPs in the region of calcaneus is a high level, causing greater development in patients with intrinsic and intrinsic facts.

Conclusion: It is hoped that the results of this research may support the development of new studies, contribute to the safety and quality of care in institutions.

Keywords: femur fracture, Immobilization scale, pressure injury, nursing, trauma, care practices, flictema, patient safety, investigation
Volume 6 Issue 5 - 2019

\author{
Adriana Fank,' Caren Leticia de Souza \\ Milani,' Graziele jacob pimenta ${ }^{2}$ \\ 'Nurse, Bachelor of Nursing from the Faculty of Biomedical \\ Sciences of Cacoal-FACIMED, Specialist in Adult and Pediatric \\ ICU Nursing, Brazi \\ ${ }^{2}$ State University of Mato Grosso, Brazil
}

Correspondence: Caren Leticia de Souza Milani, Nurse, Bachelor of Nursing from the Faculty of Biomedical Sciences of Cacoal-FACIMED, Specialist in Adult and Pediatric ICU Nursing, Brazil, Email milani.caren@hotmail.com

Received: July 26, 2019 | Published: September 205, 2019

\section{Introduction}

Fibrous fracture is one of the major public health problems. Most of the trauma is of low energetic load and is related to conditions such as malnutrition, lack of physical activity, increased visual acuity and reflexes, sarcopenia and mainly bone fragility'. In Brazil, for example, pressure lesions can be a serious public health problem, over the index of patients presenting the severity of skin disease. The overall incidence of pressure is $37.03 \%$. It should be noted that a high hypothesis is related to the risk of risk to risk to risk to risk to risk. hospitalization and is caused by directing trauma processes to the use of prolonged immobility without the use of protection is a risk factor for the presence of pressure injuries. ${ }^{1}$

The appearance of pressure injury is closely related to several factors, care processes and the environment. Being that the nurse became the figure that is next to the treatment of wounds. Thus, a broad view on the polytraumatized patient should be based on estimation zones and scientific, thus minimizing possible complications for the patient with regard to the quality of service and patient safety. ${ }^{2}$

The objective of this study was to identify and characterize the factors that influenced the appearance of pressure injury in a patient suffering from motorcycle accident at the regional hospital of Vilhena using immobilization by splint for immobilization (Made in an anti - rust coated, EVA virgin). To raise the complications caused by the use of prolonged immobilization without presence of specific protection for bony prominences and to show by means of real case complications related to the care. Indicate measures for immediate intervention in the hospital unit.

Faced with this, research became a cause of interest among nurses due to the scientific/technological progress and necessity in the field of emergency and trauma residence and the need for improved technical and scientific knowledge of the nursing professional in the evaluation of these patients, in order to intervene directly, promoting care and avoiding the development of diseases related to the use of splints, for example. We perceive that trauma is the center of learning in the emergency scenario so a better investigation allows the construction and scientific contribution of teaching and nursing care practices to patients who are victims of trauma.

\section{Case report}

A 20-year-old LSS from a public hospital located in the city of Vilhena, Rondônia, was admitted to the emergency room of the HRV on $28 \backslash 12 \backslash 2017$, where he was hospitalized due to a motorcycle accident brought by the firefighter, diagnosed with a fracture of femur and diaphyseal. The same one was in use of splint for immobilization without use of protection of the calcaneus. Presenting in the 1 day of hospitalization numbness, formation and posterior loss of sensitivity. Transferred to the good Jesus hospital to perform a surgical procedure, later hears the appearance of the flictema, and thus the patient was discharged without the detection of the pressure injury and follow-up.

After 45 days in the return with the orthopedist showed the lesion to the same one, in which it prescribed fibrase for the accomplishment of the dressing, presented lesion necrosed tissue and hospital followup for a better characterization of the lesion.

The patient's skin follow-up was given daily by the two researchers, showing the characterization and evolution of the lesion. The skin was evaluated through discussions among the researchers about dressings and materials to be used in the prevention of pressure lesions (Figure $1)$. 


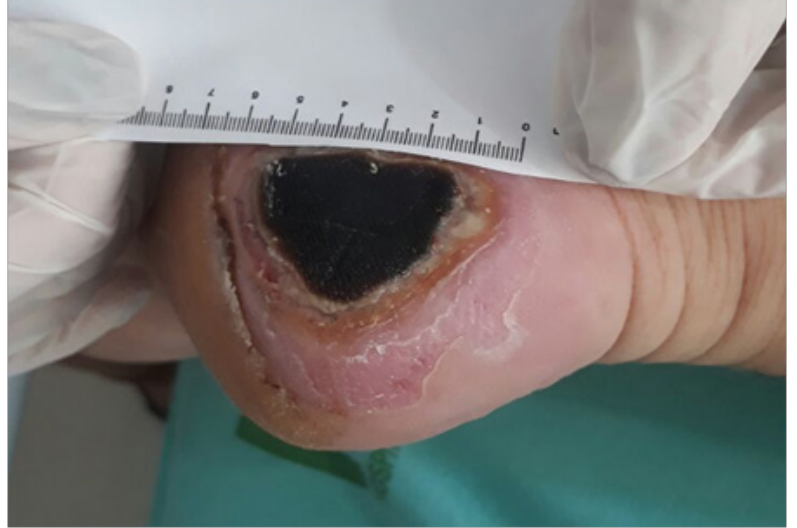

Source: personal archive

Figure I Injury in the calcaneus region.

When the lesion was identified, the initial approach of the researchers was to perform mechanical debridement with the removal of the necrotic tissue, using the first application of alginate hydrogel. The frequency of bandages was given daily for better evolution of the lesion.

After 10 days of treatment (Figure 2), it is possible to notice epithelial points with presence of tissue with shedding in the wound bed. Mechanical debridement was performed, placed in the bed of the SILVER COAT wound, on the edge of the wound with Alginate and in the distal to the bed AGE was applied.

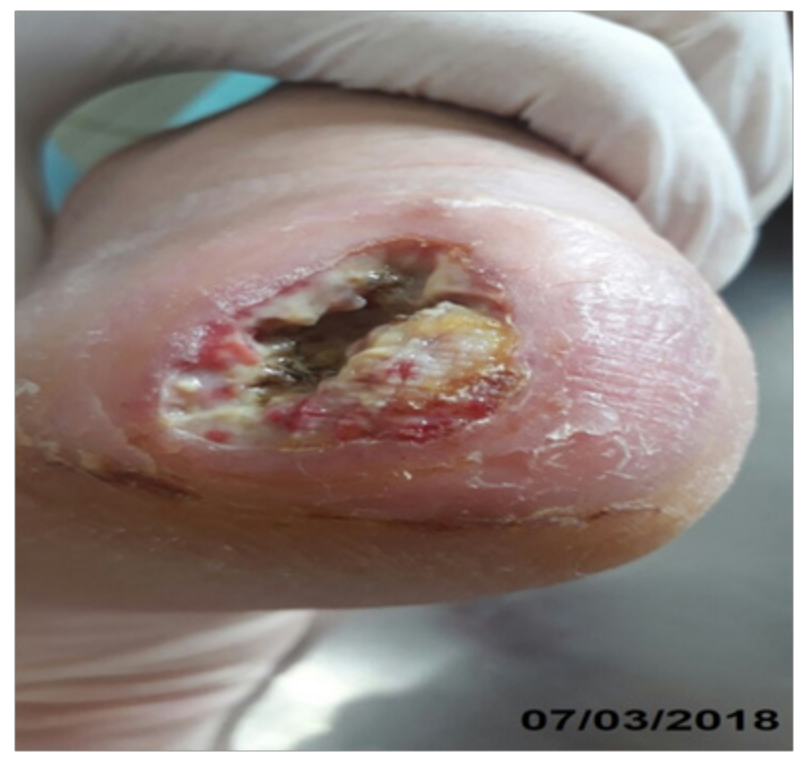

Source: personal archive

Figure 2 Lesion with sphincter-like tissue.

It was instituted by the researchers the treatment with papain $6 \%$, after the 13 day of treatment, also exerting a debriding but effective function for this type of tissue. On the 25th day, the cover SILVER IV (dressing with silver alginate) and at the edge of the AGE wound was used. Presenting epithelialization to a large extent of the lesion. Complete epithelization of the lesion occurred after sixty days of follow-up of the lesion (Figure 3).

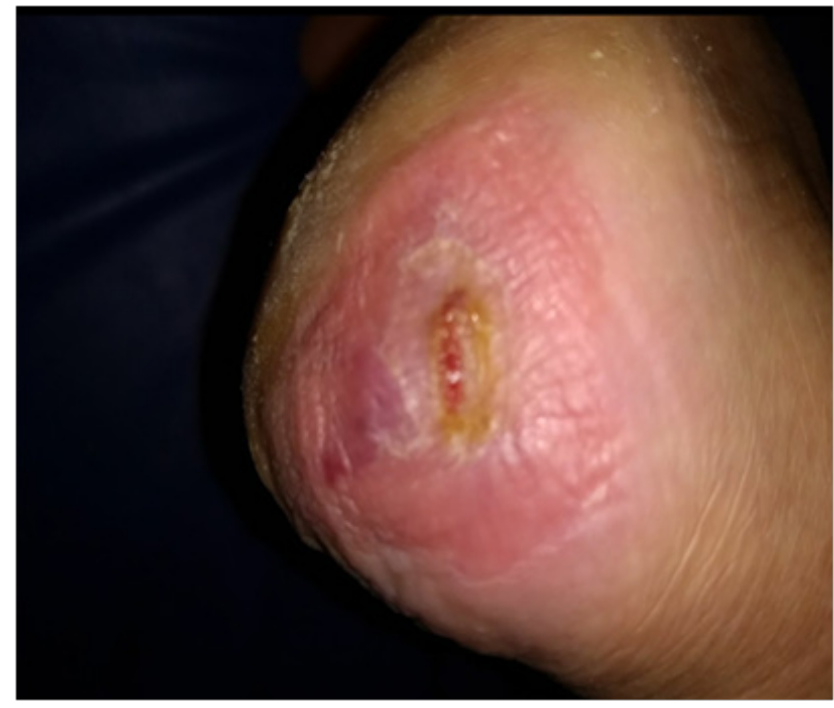

Source: personal archive

Figure 3 Complete epithelization of the lesion.

\section{Discussion}

Several factors may predispose an individual to the development of lesions. Time and pressure intensity and tissue tolerance are considered risk factors for LPP. ${ }^{3}$ In the patient under study, the type of fracture, immobilization with splint and nutritional factors stand out as risk factors. It is noted the importance of adherence to control and awareness measures of all professional classes with the population to better prevent LP in both young and old patients. ${ }^{4}$

Diagnoses of systemic arterial hypertension (SAH) and other chronic noncommunicable diseases can often affect perceptual ability, blood circulation, oxygenation, mobility, level of consciousness, and alter levels of electrolytes and proteins. In addition, they may increase the chances of LPP. ${ }^{5}$

In a study of LPP prevalence it was verified that the appearance in the calcaneal region is the second most affected site, with an incidence varying between $19.5 \%$ and $27.8 \%$. This study highlights one of the factors that promote the development of LPP in intrinsic and extrinsic factors, and friction and moisture contribute to this process. ${ }^{4,6,7}$

Lesions can develop anywhere where there are bony prominences. Lesions were found in the head, sacrococcygeal region, iliac region, upper limbs and lower limbs. The sacrococcygeal region was the most frequent, with $31.2 \%$, followed by the bilateral calcaneal region, with $25.7 \%$. The occipital region accounted for only $1.4 \%{ }^{8}$

In the patient in question, it is believed that due to the type of fracture and lack of knowledge through it, they contributed to the development of the lesion. And because with the type of immobilization increased the frequency of shearing on the skin, contributing to the vulnerability of the tissue. The force is defined as shear of traction exerted on the skin, causing it to slide over the muscular plane, which causes occlusion or rupture of the capillaries responsible for perfusion of the skin at the site. ${ }^{9}$

Regarding the treatment of lesions with dry necrosis, evidenced in this study in the region of the calcaneus, can be performed by 
means of mechanical or chemical debridement. The use of chemical debridement is a less aggressive method when compared to mechanical debridement and is often used as a treatment of choice. Evidencebased clinical practice contributes to decision-making through scientific evidence that enables recommendations for the effective use of lower cost coverage. Other reports are successfully described the use of the coverages cited in the research as a form of treatment of wound necrosis of traumatic injury by automobile accident and in occipital and calcaneal injury. ${ }^{2,8,10}$

Although this study has as a limitation the fact that it is a unique case, it is relevant for health professionals, especially nurses, for managers of the institutions and for the practice of care for people with LPP, because it seeks to analyze the effectiveness of therapeutics implemented in lesions, and should be replicated in other institutions and other patient profiles.

LPP treatment is considered a challenge for health care; the search for new technologies and products that minimize the time of therapy with efficiency should be increasingly valued in the institutions and, especially, by the nurses, since it is the professional category most professionalized for this care. Institutions need to foster research in order to test and validate methods that can produce important results for increasingly qualified assistance. ${ }^{11}$

\section{Funding details}

None.

\section{Acknowledgments}

None.

\section{Conflict of interests}

The authors declare that there is no conflict of interest.

\section{References}

1. Lima, Paula Rodrigues. Ocurrence of pressure injury in hospitalized patients: an integrative review. Revista uningá review. 2017;32(1):53-67.

2. Leite AP, Oliveira BGRB, Soares MF, et al. Use and effectiveness of papain in the wound healing process: a systematic review. Rev Gaucha Enferm. 2012;33(3):198-207.

3. Berlowitz, Dan R, Brienza, et al. Are all pressure ulcers the result of deep tissue injury? A review of the literature. Ostomy Wound Management. 2007;53(10):34-38

4. Lima AC, Tavares, Oliveira Jéssica, et al. Nursing in emergency care: risks and preventive measures of infection. Rev Nursing Electronics, Center for Nursing and Nutrition Studies, Goiás. 2011;3(3):4-6.

5. Flávia Sampaio Latini, Marisa Antonini R, Ribeiro Bastos, et al. Risk assessment for pressure ulcer in critically patients. Journal of Nursing School. 2001;45(2):313-318.

6. Luz. Sheila Rampazzo et al. Pressure ulcers. Geriatrics \& Gerontology. 2010;4(1):36-43.

7. Matos Letícia Sousa, Nalu Lopes Vasconcelos Duarte, Rita Cassia Minetto, et al. Incidence and prevalence of pressure ulcer in ICU of a Public Hospital of the Federal District. Rev. Eletr. Nurse. 2010;12(4):719-726.

8. Meire, Mariana do Valle. Treatment of pressure lesions with gel based on saline solution 20\%: case report. Revista pelle sana. 2016;3(1):2016.

9. Wada Alexandre, Neto Nuberto Teixeira, Ferreira Marcus Castro. Pressure ulcers. Journal of Medicine. 2010;89(3-4):70-177.

10. Passoni, Reginaldo. Handmade negative pressure therapy as an adjunct to cutaneous autogeny in orthopedic trauma. Journal of Nursing. 2015;5(3):580-588.

11. Neto, Agrimeron Antonio, Delmiro Santos. Fracture of female in hospitalized elderly: integrative review. Undergraduate NotebookBiological Sciences and Health-unit-alagoas. 2018;4(2):203. 